\title{
Stability Analysis of a Stochastic SIR Epidemic Model with Specific Nonlinear Incidence Rate
}

\author{
Jihad Adnani, ${ }^{1}$ Khalid Hattaf, ${ }^{1,2}$ and Noura Yousfi ${ }^{1}$ \\ ${ }^{1}$ Department of Mathematics and Computer Science, Faculty of Sciences Ben M'sik, Hassan II University, \\ P.O. Box 7955, Sidi Othman, Casablanca, Morocco \\ ${ }^{2}$ Centre Régional des Métiers de l'Education et de la Formation (CRMEF), Derb Ghalef, Casablanca, Morocco \\ Correspondence should be addressed to Jihad Adnani; adnanijihad@gmail.com and Khalid Hattaf; k.hattaf@yahoo.fr
}

Received 30 May 2013; Accepted 21 August 2013

Academic Editor: Josefa Linares-Pérez

Copyright (C) 2013 Jihad Adnani et al. This is an open access article distributed under the Creative Commons Attribution License, which permits unrestricted use, distribution, and reproduction in any medium, provided the original work is properly cited.

We investigate a stochastic SIR epidemic model with specific nonlinear incidence rate. The stochastic model is derived from the deterministic epidemic model by introducing random perturbations around the endemic equilibrium state. The effect of random perturbations on the stability behavior of endemic equilibrium is discussed. Finally, numerical simulations are presented to illustrate our theoretical results.

\section{Introduction}

Many mathematical models have been developed in order to understand disease transmissions and behavior of epidemics. One of the earliest of these models was used by Kermack and Mckendrick [1], by considering the total population into three classes, namely, susceptible $(S)$ individuals, infected $(I)$ individuals, and recovered $(R)$ individuals which is known to us as SIR epidemic model. This SIR epidemic model is very important in today's analysis of diseases.

The disease transmission process is unknown in detail. However, several authors proposed different forms of incidences rate in order to model this disease transmission process. In this paper, we consider the following model with specific nonlinear incidence rate:

$$
\begin{gathered}
\frac{d S}{d t}=A-\mu S-\frac{\beta S I}{1+\alpha_{1} S+\alpha_{2} I+\alpha_{3} S I} \\
\frac{d I}{d t}=\frac{\beta S I}{1+\alpha_{1} S+\alpha_{2} I+\alpha_{3} S I}-(\mu+d+r) I,
\end{gathered}
$$

where $A$ is the recruitment rate of the population, $\mu$ is the natural death rate of the population, $d$ is the death rate due to disease, $r$ is the recovery rate of the infective individuals, $\beta$ is the infection coefficient, and $\beta S I /\left(1+\alpha_{1} S+\alpha_{2} I+\alpha_{3} S I\right)$ is the incidence rate, where $\alpha_{1}, \alpha_{2}, \alpha_{3} \geq 0$ are constants. It is very important to note that this incidence rate becomes the bilinear incidence rate if $\alpha_{1}=\alpha_{2}=\alpha_{3}=0$, the saturated incidence rate if $\alpha_{1}=\alpha_{3}=0$ or $\alpha_{2}=\alpha_{3}=0$, the modified saturated incidence rate proposed in $[2,3]$ when $\alpha_{3}=0$, and CrowleyMartin functional response presented in [4-6] if $\alpha_{3}=\alpha_{1} \alpha_{2}$.

On the other hand, environmental fluctuations have great influence on all aspects of real life. The aim of this work is to study the effect of these environmental fluctuations on the model (1). We assume that the stochastic perturbations are of white noise type and that they are proportional to the distances of $S$ and $I$, respectively. Then, the system (1) will be extended to the following system of stochastic differential equation:

$$
\begin{aligned}
d S= & {\left[\Lambda-\mu S-\frac{\beta S I}{1+\alpha_{1} S+\alpha_{2} I+\alpha_{3} S I}\right] d t } \\
& +\sigma_{1}\left(S-S^{*}\right) d B_{1}, \\
d I= & {\left[\frac{\beta S I}{1+\alpha_{1} S+\alpha_{2} I+\alpha_{3} S I}-(\mu+d+r) I\right] d t } \\
& +\sigma_{2}\left(I-I^{*}\right) d B_{2},
\end{aligned}
$$

where $S^{*}, I^{*}$ are the positive points of equilibrium for the corresponding deterministic system (1), $B_{i}(i=1,2)$ are 
independent standard Brownian motions, and $\sigma_{i}^{2}(i=1,2)$ represent the intensities of $B_{i}$, respectively.

The rest of paper is organized as follows. In the next section, we present the stability analysis of our stochastic model (2). In Section 3, we present the numerical simulation to illustrate our result. The conclusion of our paper is in Section 4.

\section{Stability Analysis of Stochastic Model}

Clearly, the system (1) has a basic reproduction number given by

$$
R_{0}=\frac{\beta A}{\left(\mu+\alpha_{1} A\right)(\mu+d+r)} .
$$

Using the results presented by Hattaf et al. in [7], it is easy to show that if $R_{0} \leq 1$, the system (1) has just one diseasefree equilibrium $E_{f}(A / \mu, 0)$ which is globally asymptotically stable; otherwise, if $R_{0}>1$, the disease-free equilibrium $E_{f}$ is still present and is unstable, but there is also a unique positive endemic equilibrium $E^{*}\left(S^{*}, I^{*}\right)$, where $S^{*}=2 \mu\left(a+\alpha_{1} A\right) /(\beta+$ $\left.\alpha_{2} \mu-\alpha_{1} a-\alpha_{3} A+\sqrt{\delta}\right), I^{*}=\left(A-\mu S^{*}\right) / a$ with $a=\mu+d+r$, and $\delta=\left(\beta+\alpha_{2} \mu-\alpha_{1} a-\alpha_{3} A\right)^{2}+4 \alpha_{3} \mu\left(a+\alpha_{1} A\right)$. This endemic equilibrium is globally asymptotically stable.

The system (2) has the same equilibria as the system (1). We assume that $R_{0} \leq 1$, and we discuss the stability of the endemic equilibrium $E^{*}$ of (2). The stochastic system (2) can be centered at its interior endemic equilibrium $E^{*}$ by the changes of the variables as follows:

$$
u_{1}=S-S^{*}, \quad u_{2}=I-I^{*}
$$

Hence, the linearized version corresponding to the stochastic model (2) around $E^{*}$ is given by the following form:

$$
d u(t)=f(u(t)) d t+g(u(t)) d B(t)
$$

where

$$
f(u)=\left(\begin{array}{cc}
u(t)=\left(u_{1}(t), u_{2}(t)\right)^{T}, \\
\frac{\beta I^{*}\left(1+\alpha_{2} I^{*}\right)}{\left(1+\alpha_{1} S^{*}+\alpha_{2} I^{*}+\alpha_{3} S^{*} I^{*}\right)^{2}} & -\frac{\beta S^{*}\left(1+\alpha_{1} S^{*}\right)}{\left(1+\alpha_{1} S^{*}+\alpha_{2} I^{*}+\alpha_{3} S^{*} I^{*}\right)^{2}} \\
\frac{\beta I^{*}\left(1+\alpha_{2} I^{*}\right)}{\left(1+\alpha_{1} S^{*}+\alpha_{2} I^{*}+\alpha_{3} S^{*} I^{*}\right)^{2}} & \frac{\beta S^{*}\left(1+\alpha_{1} S^{*}\right)}{\left(1+\alpha_{1} S^{*}+\alpha_{2} I^{*}+\alpha_{3} S^{*} I^{*}\right)^{2}}-a
\end{array}\right) u,
$$

and the superscript " $T$ " represents transposition.

Clearly the endemic equilibrium $E^{*}$ corresponds to the trivial solution $u(t)=0$ in (5).

Let $C^{1,2}\left([0,+\infty) \times \mathbb{R}^{2} ; \mathbb{R}^{+}\right)$be the family of nonnegative functions $W(t, u)$ defined on $[0,+\infty) \times \mathbb{R}^{2}$ such that they are continuously differentiable with respect to $t$ and twice with respect to $u$. From [8], we define the differential operator $L$ for a function $W(t, u) \in C^{1,2}\left([0,+\infty) \times \mathbb{R}^{2} ; \mathbb{R}^{+}\right)$by

$$
\begin{aligned}
L W(t, u)= & \frac{\partial W(t, u)}{\partial t}+f^{T}(u) \frac{\partial W(t, u)}{\partial u} \\
& +\frac{1}{2} \operatorname{Tr}\left[g^{T}(u) \frac{\partial^{2} W(t, u)}{\partial u^{2}} g(u)\right],
\end{aligned}
$$

where $\partial W / \partial u=\left(\partial W(t, u) / \partial u_{1}, \partial W(t, u) / \partial u_{2}\right)^{T}$ and $\partial^{2} W(t$, $u) / \partial u^{2}=\left(\partial^{2} W / \partial u_{i} \partial u_{j}\right)_{i, j}$.

According to [8], we have the following theorem.
Theorem 1. Suppose that there exists a function $W(t, u) \in$ $C^{1,2}\left([0,+\infty) \times \mathbb{R}^{2} ; \mathbb{R}^{+}\right)$satisfying the following inequalities:

$$
\begin{gathered}
K_{1}|u|^{p} \leq W(t, u) \leq K_{2}|u|^{p} \\
L W(t, u) \leq-K_{3}|u|^{p}
\end{gathered}
$$

where $K_{i}, i=1,2$, and $p$ are positive constants. Then, the trivial solution of (5) is exponentially p-stable for $t \geq 0$. Moreover, if $p=2$, then the trivial solution is also called asymptotically mean square stable and it is globally asymptotically stable in probability.

From Theorem 1, we get the conditions for stochastic asymptotic stability of trivial solution of (5) which are given by the following theorem.

Theorem 2. Assume that $R_{0}>1, \sigma_{1}^{2} \leq 2\left[\mu+\left(\beta I^{*}(1+\right.\right.$ $\left.\left.\left.\alpha_{2} I^{*}\right) /\left(1+\alpha_{1} S^{*}+\alpha_{2} I^{*}+\alpha_{3} S^{*} I^{*}\right)^{2}\right)\right]$, and $\sigma_{2}^{2} \leq 2\left(\alpha_{2} I^{*}+\right.$ $\left.\alpha_{3} S^{*} I^{*}\right) a /\left(1+\alpha_{1} S^{*}+\alpha_{2} I^{*}+\alpha_{3} S^{*} I^{*}\right)$ hold. Then, the trivial solution of (5) is asymptotically mean square stable. 
Proof. We consider the following Lyapunov function:

$$
W(t, u)=\frac{1}{2}\left(w_{1} u_{1}^{2}+w_{2} u_{2}^{2}\right)
$$

where $w_{1}$ and $w_{2}$ are nonnegative constants that will be chosen later. It is easy to verify that inequality (8) holds true with $p=2$.

By applying the operator $L$ on $W(t, u)$, we get

$$
\begin{aligned}
L W(t, u)= & -\left[\mu+\frac{\beta I^{*}\left(1+\alpha_{2} I^{*}\right)}{\left(1+\alpha_{1} S^{*}+\alpha_{2} I^{*}+\alpha_{3} S^{*} I^{*}\right)^{2}}\right] w_{1} u_{1}^{2} \\
& -\frac{\beta S^{*}\left(1+\alpha_{1} S^{*}\right)}{\left(1+\alpha_{1} S^{*}+\alpha_{2} I^{*}+\alpha_{3} S^{*} I^{*}\right)^{2}} w_{1} u_{1} u_{2} \\
& +\frac{\beta I^{*}\left(1+\alpha_{2} I^{*}\right)}{\left(1+\alpha_{1} S^{*}+\alpha_{2} I^{*}+\alpha_{3} S^{*} I^{*}\right)^{2}} w_{2} u_{2} u_{1} \\
& -\left[a-\frac{\beta S^{*}\left(1+\alpha_{1} S^{*}\right)}{\left(1+\alpha_{1} S^{*}+\alpha_{2} I^{*}+\alpha_{3} S^{*} I^{*}\right)^{2}}\right] w_{2} u_{2}^{2} \\
& +\frac{1}{2} \operatorname{Tr}\left[g^{T}(u) \frac{\partial^{2} W(t, u)}{\partial u^{2}} g(u)\right] \\
= & -\left[\mu+\frac{\beta I^{*}\left(1+\alpha_{2} I^{*}\right)}{\left(1+\alpha_{1} S^{*}+\alpha_{2} I^{*}+\alpha_{3} S^{*} I^{*}\right)^{2}}-\frac{1}{2} \sigma_{1}^{2}\right] \\
& \times w_{1} u_{1}^{2}
\end{aligned}
$$$$
B=\left(\begin{array}{c}
{\left[\mu+\frac{\beta I^{*}\left(1+\alpha_{2} I^{*}\right)}{\left(1+\alpha_{1} S^{*}+\alpha_{2} I^{*}+\alpha_{3} S^{*} I^{*}\right)^{2}}-\frac{1}{2} \sigma_{1}^{2}\right] w_{1}} \\
0
\end{array}\right.
$$

From the assumptions of the theorem, we deduce that $b_{i i}>$ $0, i=1,2$ and $|B|>0$. Hence, $B$ is a symmetric positive definite matrix. Let $\lambda_{m}$ denote the minimum of its two positive eigenvalues $\lambda_{1}$ and $\lambda_{2}$; then, we can easily get

$$
L W(t, u) \leq-\lambda_{m}|u|^{2} .
$$

According to Theorem 1, we conclude that the trivial solution of system (5) is globally asymptotically stable.

\section{Numerical Simulations}

In this section, we present the numerical simulations to illustrate our theoretical results.

We use the following parameter values: $A=0.9, \mu=0.1$, $\beta=0.5, d=0.1, r=0.1, \alpha_{1}=0.1, \alpha_{2}=0.1, \alpha_{3}=0.01$, $\sigma_{1}=0.1$, and $\sigma_{2}=0.01$. In this case, we have $R_{0}=7.8947>1$, $\sigma_{1}^{2}=0.01<2.4539$, and $\sigma_{2}^{2}=0.0001<0.00033502$. By applying Theorem 2, we deduce that the endemic equilibrium$$
\left.\left[\frac{\left(\alpha_{2} I^{*}+\alpha_{3} S^{*} I^{*}\right) a}{1+\alpha_{1} S^{*}+\alpha_{2} I^{*}+\alpha_{3} S^{*} I^{*}}-\frac{1}{2} \sigma_{2}^{2}\right] w_{2}\right) .
$$

$$
\begin{aligned}
& -\left[\frac{\beta S^{*}\left(1+\alpha_{1} S^{*}\right)}{\left(1+\alpha_{1} S^{*}+\alpha_{2} I^{*}+\alpha_{3} S^{*} I^{*}\right)^{2}} w_{1}\right. \\
& \left.\quad-\frac{\beta I^{*}\left(1+\alpha_{2} I^{*}\right)}{\left(1+\alpha_{1} S^{*}+\alpha_{2} I^{*}+\alpha_{3} S^{*} I^{*}\right)^{2}} w_{2}\right] u_{1} u_{2} \\
& -\left[a-\frac{\beta S^{*}\left(1+\alpha_{1} S^{*}\right)}{\left(1+\alpha_{1} S^{*}+\alpha_{2} I^{*}+\alpha_{3} S^{*} I^{*}\right)^{2}}-\frac{1}{2} \sigma_{2}^{2}\right] \\
& \times w_{2} u_{2}^{2} .
\end{aligned}
$$

If we choose $w_{1} S^{*}\left(1+\alpha_{1} S^{*}\right)=w_{2} I^{*}\left(1+\alpha_{2} I^{*}\right)$, then

$$
\begin{aligned}
L W(t, u)=- & {\left[\mu+\frac{\beta I^{*}\left(1+\alpha_{2} I^{*}\right)}{\left(1+\alpha_{1} S^{*}+\alpha_{2} I^{*}+\alpha_{3} S^{*} I^{*}\right)^{2}}-\frac{1}{2} \sigma_{1}^{2}\right] } \\
& \times w_{1} u_{1}^{2} \\
& -\left[a-\frac{\beta S^{*}\left(1+\alpha_{1} S^{*}\right)}{\left(1+\alpha_{1} S^{*}+\alpha_{2} I^{*}+\alpha_{3} S^{*} I^{*}\right)^{2}}-\frac{1}{2} \sigma_{2}^{2}\right] \\
& \times w_{2} u_{2}^{2} \\
= & -u^{T} B u,
\end{aligned}
$$

where

$E^{*}$ is globally asymptotically stable. Figure 1 demonstrates the above analysis.

\section{Conclusion}

The purpose of this work is to study the effects of the environmental fluctuations on dynamical behavior of a deterministic SIR epidemic model with specific nonlinear incidence rate by considering the white noise perturbation around the endemic equilibrium state. We have shown that our stochastic model is globally asymptotically stable in probability when the intensities of white noise are less than certain threshold of parameters. However, if these intensities of white noise are zero, which meant that there is no environmental stochastic perturbation, then the conditions of Theorem 2 are reduced to the condition $R_{0}>1$, which gives a nonlinear stability condition for the deterministic model (1).

From our analytical and numerical results, we conclude that the main factor that affects the stability of the stochastic 


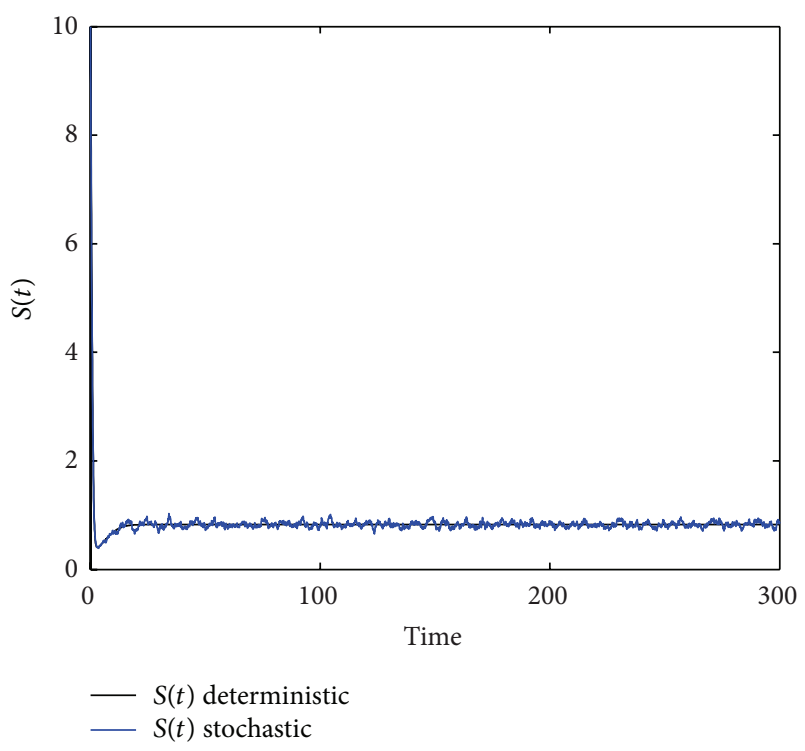

(a)

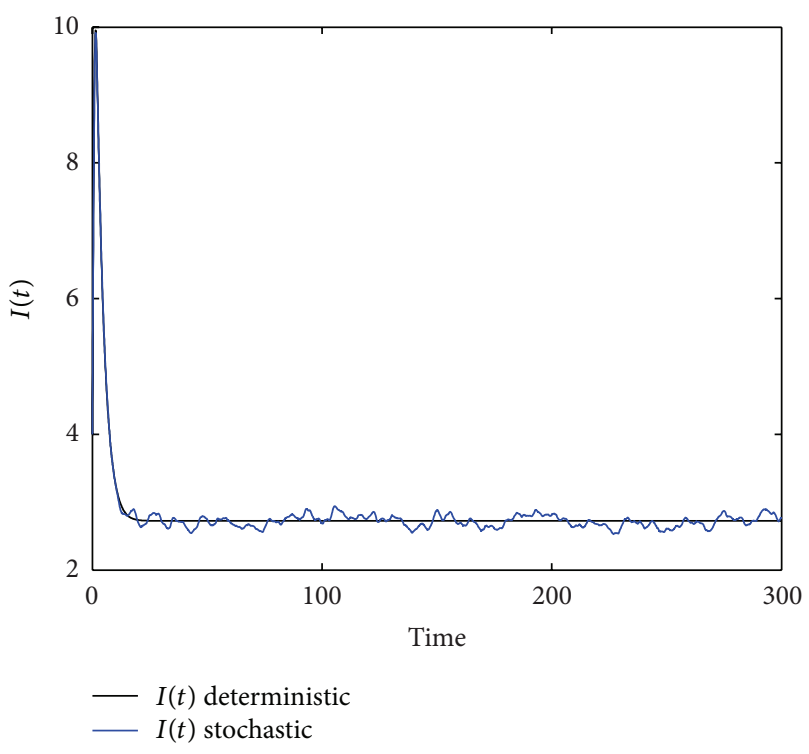

(b)

FIGURE 1: Deterministic and stochastic trajectories of models (1) and (2) with parameter values $A=0.9, \mu=0.1, \beta=0.5, d=0.1, r=0.1$, $\alpha_{1}=0.1, \alpha_{2}=0.1, \alpha_{3}=0.01, \sigma_{1}=0.1$, and $\sigma_{2}=0.01$.

model is the intensities of white noise. In addition, our main results extend the corresponding results in paper [3] and those in [9] when the value of the parameter ' $h$ ' is equal to one into the stochastic model [9].

\section{References}

[1] W. O. Kermack and A. G. McKendrick, "Contributions to the mathematical theory of epidemics," Proceedings of the Royal Society A: Mathematical, Physical and Engineering Sciences, vol. 115, pp. 700-721, 1927.

[2] A. Kaddar, "On the dynamics of a delayed sir epidemic model with a modified saturated incidence rate," Electronic Journal of Differential Equations, vol. 2009, no. 133, pp. 1-7, 2009.
[3] P. Das, D. Mukherjee, and Y. H. Hsieh, "An S-I epidemic model with saturation incidence: discrete and stochastic version," International Journal of Nonlinear Analysis and Applications, pp. $1-9,2011$.

[4] P. H. Crowley and E. K. Martin, "Functional responses and interference within and between year classes of a dragony population," Journal of the North American Benthological Society, vol. 8, pp. 211-221, 1989.

[5] X. Zhou and J. Cui, "Global stability of the viral dynamics with crowley-martin functional response," Bulletin of the Korean Mathematical Society, vol. 48, no. 3, pp. 555-574, 2011.

[6] X. Q. Liu, S. M. Zhong, B. D. Tian, and F. X. Zheng, "Asymptotic properties of a stochastic predator-prey model with CrowleyMartin functional response," Journal of Applied Mathematics and Computing, 2013.

[7] K. Hattaf, A. A. Lashari, Y. Louartassi, and N. Yousfi, "A delayed SIR epidemic model with general incidence rate," Electronic Journal of Qualitative Theory of Differential Equations, vol. 3, pp. $1-9,2013$.

[8] V. N. Afanas'ev, V. B. Kolmanowskii, and V. R. Nosov, Mathematical Theory of Control Systems Design, Kluwer Academic, Dordrecht, Netherlands, 1996.

[9] P. Das, D. Mukherjee, and A. K. Sarkar, "Study of an S-I epidemic model with nonlinear incidence rate: discrete and stochastic version," Applied Mathematics and Computation, vol. 218, no. 6, pp. 2509-2515, 2011. 


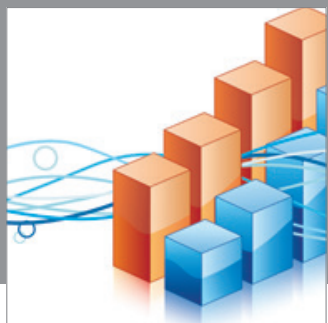

Advances in

Operations Research

mansans

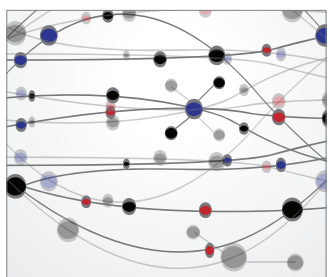

The Scientific World Journal
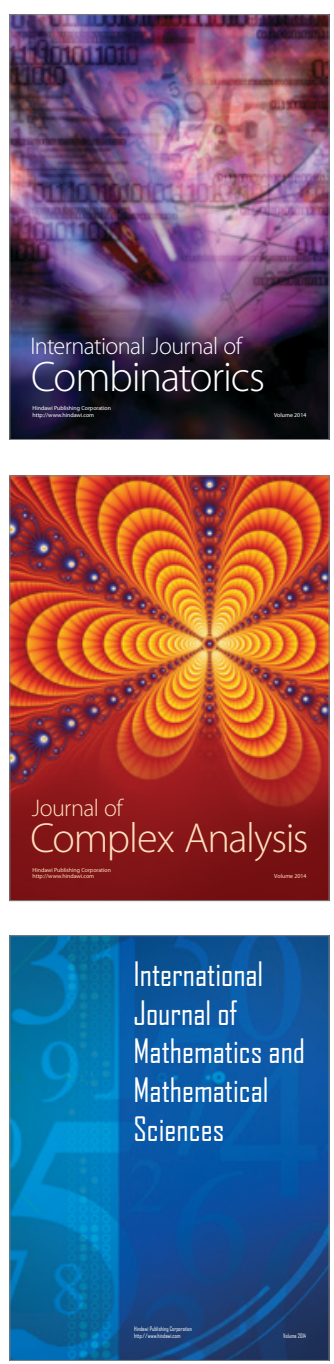
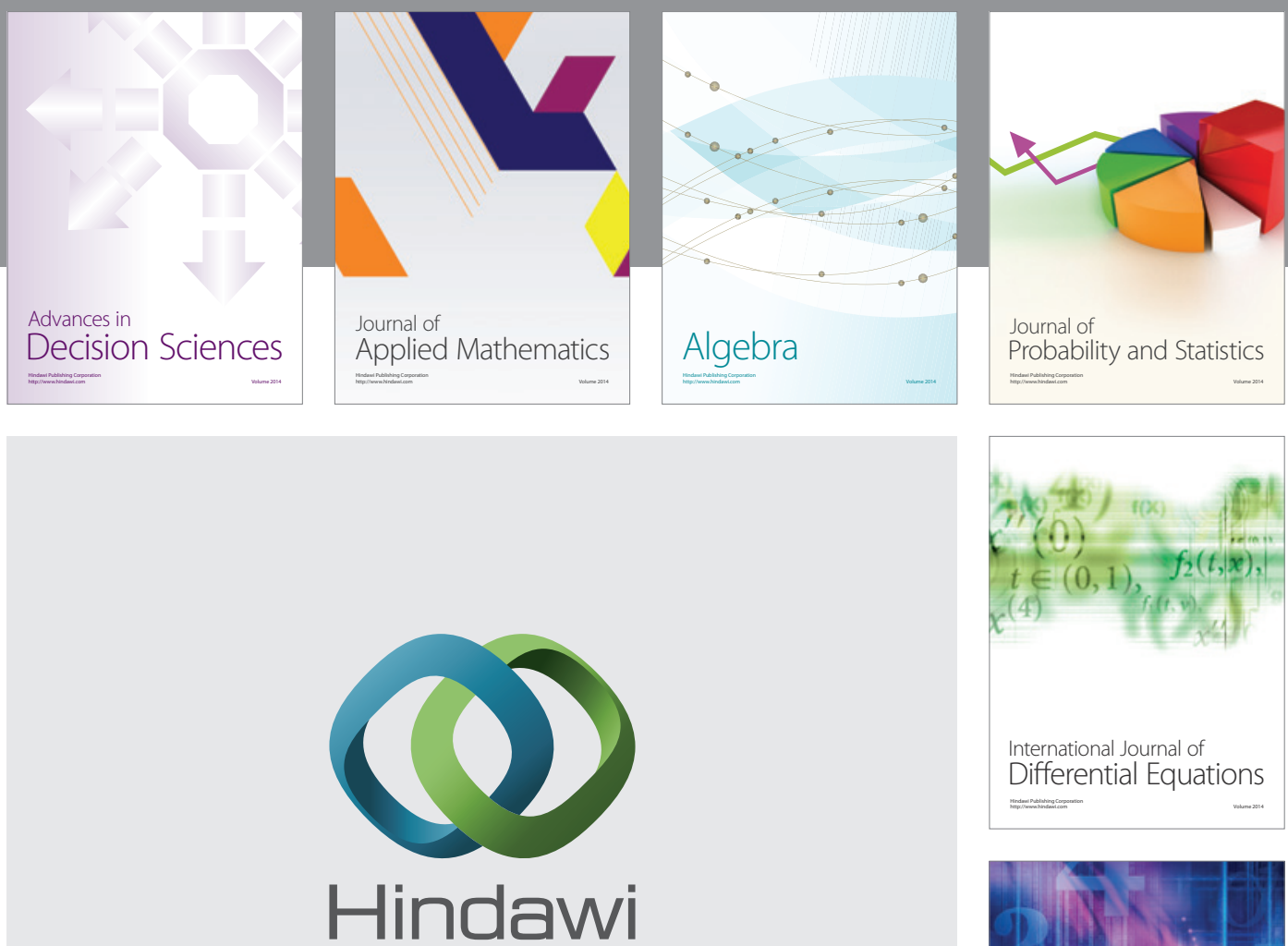

Submit your manuscripts at http://www.hindawi.com
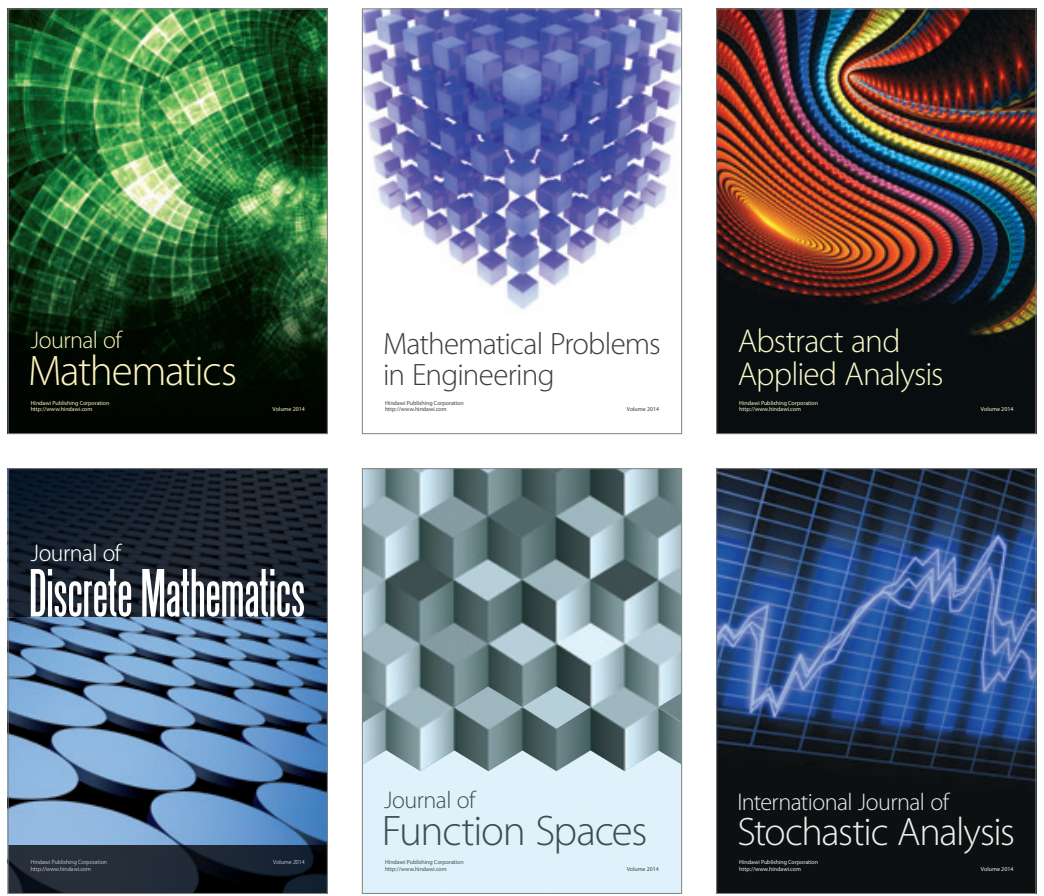

Journal of

Function Spaces

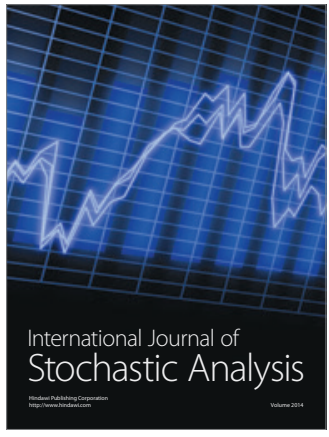

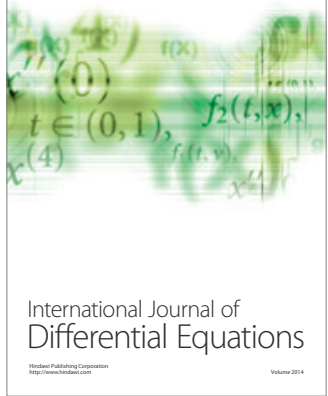
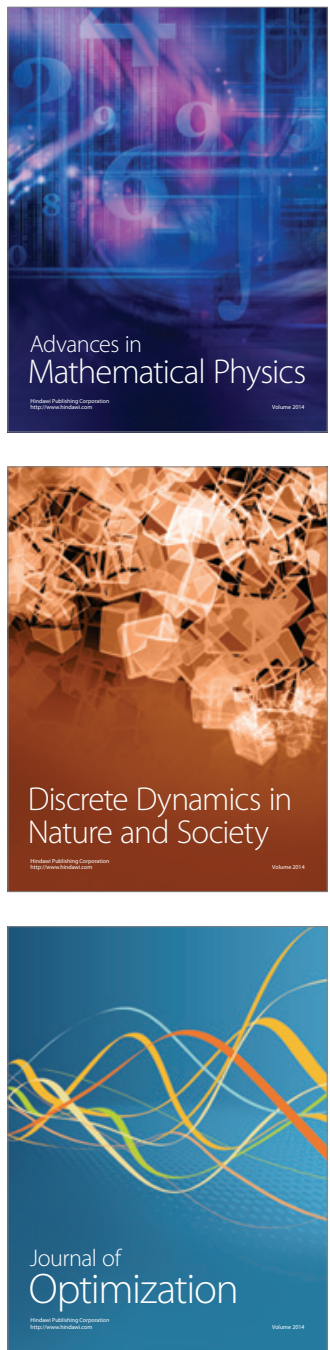